\title{
Feasibility of an exercise intervention for fatigued breast cancer patients at a community-based cardiac rehabilitation program
}

This article was published in the following Dove Press journal:

Cancer Management and Research

10 February 2017

Number of times this article has been viewed

\author{
Stefanie De Jesus' \\ Lyndsay Fitzgeorge ${ }^{2}$ \\ Karen Unsworth ${ }^{3}$ \\ David Massel ${ }^{4,5}$ \\ Neville Suskin ${ }^{4,5}$ \\ Harry Prapavessis' \\ Michael Sanatani ${ }^{6}$
}

'Department of Kinesiology, The University of Western Ontario,

${ }^{2}$ School of Health Sciences,

Fanshawe College, ${ }^{3}$ Cardiac

Rehabilitation \& Secondary

Prevention Program, St. Joseph's

Hospital, ${ }^{4}$ Division of Cardiology \&

Program of Experimental Medicine,

Schulich School of Medicine and

Dentistry, University of Western

Ontario, ${ }^{5}$ Lawson Health Research

Institute, ${ }^{6}$ Department of Medical

Oncology, Schulich School of Medicine

and Dentistry, University of Western

Ontario, London, ON, Canada
Correspondence: Michael Sanatani Department of Medical Oncology, Schulich School of Medicine and Dentistry, University of Western Ontario, 790 Commissioners Rd East, London, ON N6A4L6, Canada

Tel + I 5196858640

Fax + I 5196858624

Email Michael.Sanatani@Lhsc.on.ca
Purpose: Exercise is beneficial to quality of life after cancer treatment, yet few cancer survivors meet exercise guidelines. Our study sought to determine the feasibility of an oncology rehabilitation exercise program embedded within a cardiac rehabilitation program.

Methods: Patients who rated their fatigue $>4 / 10$ after completion of adjuvant chemotherapy for breast cancer were screened for eligibility and the outcomes were assessed (Piper Fatigue Scale, Functional Assessment of Cancer Therapy-Breast [FACT-B], Edmonton Symptom Assessment System, body composition, stress test, and physical activity measurement [accelerometer]). Participants received individualized exercise prescription. Following the 16-week program, repeat assessment plus patient acceptance and satisfaction survey was completed The primary end point was the composite of accrual rate $>25 \%$, program adherence $>80 \%$, and mean compliance with accelerometer use $>80 \%$.

Results: Twenty of 24 screened patients consented to the study and completed the baseline assessment. Adherence was 30.3\%. Mean accelerometer use was 3.88/7 days (78\%). Fatigue at baseline was rated at 4.82/10, and at $3.59(p=0.09)$ after the intervention. Overall well-being (FACT-B) score changed from 92.7 to $98.3(p=0.05)$. There were no significant changes in body composition (except for bone mineral content), aerobic exercise capacity, or activity patterns.

Conclusion: Although the primary outcome was not met, our study indicates that an oncology exercise rehabilitation program can be incorporated into an existing cardiac rehabilitation program. Based on feedback received, we propose that in order to achieve exercise goals, frequent, encouraging, and tailored feedback and group sessions to foster a sense of community may additionally be needed to strengthen adherence to a prescribed exercise program.

Keywords: breast cancer rehabilitation, fatigue, exercise, community setting

\section{Introduction}

Breast cancer is the most prevalent cancer diagnosis among women worldwide., ${ }^{1,2}$ Owing to significant improvements to early detection and biomedical treatments, breast cancer mortality rates have progressively declined. ${ }^{3}$ However, cancer-related fatigue, whether stemming primarily from treatment or directly due to the illness, is a common symptom, which is difficult to treat without motivating patients to exercise.

Extensive literature supporting the role of exercise as a non-pharmacological approach to ameliorating cancer-related fatigue has been summarized in a recent Cochrane review by Cramp and Daniel. ${ }^{4}$ Fifty-six randomized controlled trials $(\mathrm{N}=4068)$ were identified, half of which were carried out in individuals with breast cancer. This meta-analysis showed significant improvements in fatigue levels following 
an exercise intervention compared to control (standardized mean difference $-0.27,95 \%$ confidence interval -0.37 to $-0.17)$. The benefits associated with exercise were evident for interventions delivered during or post-adjuvant cancer therapy and for interventions involving aerobic exercise. In addition, exercise has been shown to have a positive effect on other psychological and physical sequelae associated with breast cancer including health-related quality of life and disease recurrence..$^{5-7}$

Nevertheless, exercise initiation and adherence are disrupted by cancer diagnosis and treatment. ${ }^{8,9}$ Bourke et al ${ }^{10}$ systematically reviewed 14 randomized controlled trials $(\mathrm{N}$ $=648$ ) that evaluated an exercise intervention versus standard care among sedentary cancer patients while addressing the question of motivation and behavior modification regarding exercise. Due to the wide variation of exercise interventions and outcomes studied, the authors chose a published adherence rate of $75 \%$ of the prescribed exercise program as an indicator of a successful implementation of an exercise program. None of the included studies were able to document such adherence at any longer term follow-up, and only three studies were able to demonstrate $>75 \%$ adherence during the exercise intervention itself. The most successful studies had included both supervised and independent exercise components. Subsequently, the BEAT study, ${ }^{11}$ which used a combination of group counseling, individual counseling, and a gradual transition from group to independent exercise, achieved a $98 \%$ adherence during the program, and $72 \%$ at 3 months.

Based on this knowledge, in order for cancer survivors (which we understand to mean any patient living with cancer or after the treatment of cancer) to benefit from the aforementioned, well-documented value of exercise on a larger scale, two aspects have to be considered. First, exercise must be adopted by the patients as part of their daily routine. Second, cancer exercise programs must be accessible outside the context of a clinical trial.

Owing to high interest levels among cancer survivors to engage in health promotion behaviors, the LIVESTRONG ${ }^{\mathrm{TM}}$ Foundation and YMCA (of the US) codeveloped Exercise and Thrive, a 12-week exercise program to facilitate the realization of health-related goals of cancer survivors (ie., off treatment). Individuals received a YMCA membership and participated in a group-based, supervised, individualized resistance training program. ${ }^{12}$ Researchers reported high adherence rates to this community-based exercise program $(88 \%)$ and low attrition ( $85 \%$ had baseline and post-program data), and consequently, physiological (ie., blood pressure, upper and lower body strength, flexibility, musculoskeletal symptoms) and psychosocial (ie., quality of life, social support, fatigue, insomnia) benefits from participating.

In a similar study, Cheifetz et al ${ }^{13}$ evaluated a community-based exercise and education program with theoretical underpinnings through a multiagency collaboration (ie., university, acute care hospital, and YMCA). Individuals at any stage of the cancer continuum were required to purchase a YMCA membership in order to participate in the 12-week supervised, multicomponent (eg., cardiovascular, strength, and flexibility training) exercise and education program. Irrespective of the high dropout rate (56\%), significant improvements were observed for exercise capacity, quality of life, and disease burden.

More recently, Dittus et $\mathrm{al}^{14}$ have leveraged an existing cardiac rehabilitation model, for which exercise is a cornerstone, as a pathway to establish oncology rehabilitation. Individuals living with cancer participated in a 12-week, supervised aerobic and resistance training program at a university-based facility. Approximately three-quarters of participants completed the program. While not communitybased, they demonstrated that cardiac rehabilitation is an ideal and cost-effective framework to provide oncology rehabilitation programs, as these facilities are often underutilized, have appropriate equipment and expertise (ie., human resources), and have the capacity to reach a wide population.

Against this background, our feasibility study sought to explore the feasibility of using a preexisting community-based cardiac rehabilitation program (ie., YMCA) to address barriers that cancer survivors encounter. This research differed from the aforementioned studies in two ways. First, given that cancerrelated fatigue profoundly affects the majority of the cancer patient population, ${ }^{15}$ only breast cancer patients reporting moderate-to-high fatigue levels were enrolled. Second, this study addressed the question of feasibility in a more comprehensive manner than other studies. Accordingly, the primary outcome of our study was the feasibility of this approach, which we defined as a composite end point including enrollment rate, attendance rate, and Actical ${ }^{\circledR}$ accelerometer data capture (MiniMitter, Bend, OR, USA), as outlined in the following section. The effect of exercise on patients' fatigue levels, quality of life, symptom severity, body composition, aerobic fitness, and physical activity levels was also assessed as secondary outcomes.

\section{Methods}

Twenty female patients were recruited from the London Regional Cancer Program to participate in this single-arm 
study. Patients were eligible to participate if they met the following criteria:

1. They had a diagnosis of invasive adenocarcinoma of the breast (stages I-III) and were treated with curative intent. If they were treated with adjuvant intravenous systemic therapy (including trastuzumab if applicable) or radiation, they had to be within 4 months (16 weeks) of the completion of such therapy. They could remain on hormonal adjuvant therapy on study.

2. They rated their fatigue level as $\geq 4 / 10$ on the clinic selfreport form.

3. They were between 18 and 55 years of age.

4. They had a baseline cardiologist assessment confirming fitness for physical exercise.

Exclusion criteria included: 1) medical or musculoskeletal comorbidities precluding participation in light-to-moderate aerobic exercise; 2) participation in moderate-to-vigorous exercise most days of the week; and 3) untreated reversible causes of fatigue including the following: hemoglobin $<100$ $\mathrm{g} / \mathrm{L}$; abnormal thyroid function (thyroid-stimulating hormone outside normal limits); untreated adrenal insufficiency; chronic renal failure with estimated creatinine clearance $<30$ $\mathrm{mL} / \mathrm{min}$; active infection or neutropenic fever; decompensated congestive heart failure; uncontrolled pulmonary disease (exercise-induced asthma not preventable with $\beta$-agonist inhalers pre-exercise, COPD with forced expiratory volume in 1 second $<1.5 \mathrm{~L}$, resting hypoxia requiring oxygen to maintain oxygen saturation $>92 \%$ ); untreated depression; hypophosphatemia $(<0.80 \mathrm{mmol} / \mathrm{L})$ or hypomagnesemia ( $<0.7 \mathrm{mmol} / \mathrm{L})$; hypercalcemia; Eastern Cooperative Oncology Group performance status 3 or 4 ; or pregnancy or lactation.

\section{Primary outcome measures}

The primary outcome of our study was the feasibility of the exercise program, which we defined as the achievement of $>25 \%$ of referred patients enrolling (accrual rate), a mean adherence rate of $>80 \%$ at the exercise sessions, and a data capture compliance rate of $>80 \%$ of the Actical ${ }^{\circledR}$ accelerometer readings at follow-up (post-intervention). Adherence to the 16-week exercise program was calculated as the ratio of the number of exercise sessions attended to the total number of exercise sessions offered, as self-reported by patients in their exercise workbook. Compliance was defined as wearing the Actical ${ }^{\circledR}$ with data capture of at least 5 out of 7 days and 10 hours each day. ${ }^{16}$

\section{Secondary outcome measures Fatigue}

Fatigue was measured by the revised Piper Fatigue Scale and has been validated in a group of breast cancer survivors. ${ }^{17}$ This instrument consists of 22 items reported on a 10-point scale (1: no distress, 10: a great deal of distress) to determine four dimensions of subjective fatigue (behavioral/severity, affective meaning, sensory, and cognitive/mood) and overall fatigue. Subscale and total fatigue scores were derived by a mean score of the respective items. In the current sample, the internal consistency was excellent at baseline for behavioral/ severity ( $\alpha=0.97)$, affective meaning ( $\alpha=0.98)$, sensory $(\alpha=0.96)$, and cognitive/mood $(\alpha=0.91)$. Behavioral/severity ( $\alpha=0.97)$, affective meaning ( $\alpha=0.95)$, sensory $(\alpha=0.98)$, and cognitive/mood $(\alpha=0.90)$ subscales were also reliable at follow-up.

\section{Quality of life}

The Functional Assessment of Cancer Therapy-Breast $(\text { FACT-B })^{18}$ was used to assess quality of life. This inventory has well-established validity and reliability. ${ }^{18,19}$ It is composed of 37 items that represent five subscales: physical well-being, social/family well-being, emotional well-being, functional well-being, and breast cancer-related well-being. Patients responded using a 5-point scale from 0 (not at all), 2 (somewhat) to 4 (very much), with higher scores indicating superior quality of life. The FACT-B scoring template was followed to determine subscale and total scores. The FACT-B demonstrated good-to-excellent internal consistency at baseline for physical $(\alpha=0.85)$, social/family ( $\alpha=0.87)$, emotional $(\alpha=0.77)$, and functional $(\alpha=0.87)$ well-being. The reliability of the FACT-B at follow-up was also acceptable to excellent: physical $(\alpha=0.84)$, social/ family ( $\alpha=0.87)$, emotional $(\alpha=0.66)$, and functional $(a=0.84)$ well-being.

\section{Symptom severity}

Symptom burden (eg., anorexia, pain, and appetite) was examined with the Edmonton Symptom Assessment System (ESAS). ${ }^{20}$ Using an 11-point numerical scale (0: symptom absence, 1-3: mild, 4-6: moderate, 7-10: severe, 11: worst possible symptom severity), nine items (ie, various symptoms) were individually profiled. The ESAS has been found to be a valid and reliable cancer patient assessment tool. ${ }^{21}$ This measure demonstrated excellent reliability at baseline $(\alpha=0.95)$ and follow-up $(\alpha=0.94)$. 


\section{Patient satisfaction}

As the community setting is a key feature of this feasibility study, an instrument developed by Hooker et al, ${ }^{22}$ used in several similar studies since, was used to examine patient satisfaction. Patients responded to 11 items using a 5-point scale (1: strongly agree; 3 : somewhat agree; 5 : strongly disagree) following the exercise program. Consistency was found to be acceptable at follow-up ( $\alpha=0.86)$.

\section{Anthropometry}

Weight was collected (Health-o-meter Professional, Pelstar $500 \mathrm{KL}$ ) after asking patients to remove their shoes and heavy clothing and was recorded to the nearest 10th of a kilogram.

\section{Body composition}

A trained technician used dual X-ray absorptiometry (iDXA; General Electric-Lunar iDXA, Ames Medical iDEXA; enCORE 2007 software version 11.40.004; Prodigy, Waukesha, WI, USA) to measure fat mass, lean mass, percent body and android fat, visceral adipose tissue, and bone mineral content (BMC). Lunar iDXA has been previously validated $^{23}$ and offers significantly better precision than previous systems. ${ }^{24}$

\section{Aerobic fitness}

Aerobic fitness was assessed by a trained exercise physiologist using a standard maximal incremental exercise protocol (ie., Bruce protocol) on a treadmill with continuous 12-lead electrocardiogram (ECG) and staged hemodynamic monitoring..$^{25,26}$ Patients' birth date, height, weight, sex, and mask size were inputted into the Quark b2 computer software, which has been validated and shown to be a reliable instrument for the purpose of measuring cardiorespiratory and metabolic variables. ${ }^{27,28}$

Peak oxygen consumption (peak $\mathrm{VO}_{2}$ ) was determined by taking the highest values during a 15 -second period and expressed in relative $(\mathrm{mL} / \mathrm{kg} / \mathrm{min})$ units. The visual analog Borg Rating of Perceived Exertion (RPE) scale ${ }^{29}$ was administered at the termination of the maximum fitness test.

\section{Physical activity}

Physical activity was objectively assessed using the Actical ${ }^{\circledR}$, which is a small lightweight $(17 \mathrm{~g})$ and water-resistant omnidirectional accelerometer. The Actical ${ }^{\circledR}$ has been shown to be a valid and reliable predictor of energy expenditure in adults. ${ }^{30}$

Patients were instructed to wear the device on the right hip during the waking hours of a 7-day period. Patients were unaware of their data while the device was in their possession. Data were collected at 15 -second epochs and converted to 1-minute epochs for data analysis. ${ }^{31}$ For complete measurement compliance, patients were required to provide a minimum of 10 hours per day for at least 5 days. ${ }^{32}$

\section{Exercise program}

At a central community-based exercise facility (YMCA of Western Ontario), patients engaged in an oncology rehabilitation exercise program embedded within a cardiac rehabilitation program which involved primarily cardiovascular exercise (cycle ergometer, treadmill, or stepper) three times per week. Patients were provided with an individualized exercise prescription for the duration of the program, which was derived from their baseline aerobic fitness (peak $\mathrm{VO}_{2}$ ) performance. During weeks $1-4$, patients were asked to exercise at $50 \%$ of their maximal oxygen consumption, which progressed to $60 \%$ during weeks $5-8$ and $70 \%$ during weeks $9-16 .{ }^{33}$ Exercise duration began at 15 minutes for weeks $1-3$ and increased by 5 minutes every week afterwards until the duration of 45 minutes was reached. Participants were at liberty to choose which modality of cardiovascular exercise (ie., cycle ergometer, treadmill, or stepper) they engaged in, so long as they worked towards their exercise prescription. Heart rate was monitored during exercise sessions using Polar heart rate monitors, and trained cardiac rehabilitation kinesiologists supervised the patients and encouraged them to achieve their targets at the YMCA. Workbooks were given to patients to self-monitor their facility-based exercise sessions and log their heart rate, exercise duration, and RPE. ${ }^{29}$

\section{Procedure}

Patients were screened for eligibility based on fatigue level as part of the usual assessment done at each cancer clinic visit which includes the ESAS fatigue subscale routinely. Posters reminding oncologists and nurses of the study eligibility criteria were posted in the cancer clinics where breast cancer patients were seen. Oncologists identified eligible patients who indicated $\geq 4 / 10$ fatigue levels. Eligible patients were referred to a cardiologist for a medical and cardiac evaluation regarding suitability for exercise and to rule out reversible causes of fatigue. Following this assessment, interested patients were invited to participate in the study and provided written consent. All procedures performed in studies involving human participants were in accordance with the ethical standards of and approved by the Human Subject Research Ethics Board, The University of Western Ontario, London, ON, Canada.

Prior to initiating the exercise program, baseline testing was completed at the Exercise and Health Psychology Laboratory (EHPL) in London, ON, Canada. During this visit, patients were asked to complete a demographic questionnaire, 
the Piper Fatigue Scale, FACT-B, and ESAS. Anthropometry, body composition, and aerobic fitness capacity were evaluated next. Patients were sent home with an accelerometer and provided verbal and written instructions regarding usage and measurement compliance. At the next scheduled cardiac rehabilitation session at the YMCA, participants in our study were invited to attend and proceeded with the exercise protocol specific to this study. The kinesiology study assistant communicated with the participants during the study and afterwards regarding scheduling.

As soon as possible following the completion of the 16-week exercise program, the aforementioned patientreported outcomes, and anthropometry, body composition, and aerobic fitness were evaluated. Patient acceptance and satisfaction with the exercise program were also assessed. Finally, patients were asked to wear the accelerometer for 1 week and were reminded of usage and compliance instructions.

\section{Statistical analyses}

This was an exploratory single-arm feasibility study, so a formal sample size calculation was not performed. However, other analogous studies, ${ }^{34,35}$ using the same instruments, also used similar sample sizes based on the psychometric properties and sensitivities of the questionnaires (Piper Fatigue Scale).

Secondary outcome measures including patient-reported outcomes, anthropometry, body composition, aerobic fitness, and physical activity levels were compared between baseline and follow-up in an exploratory analysis using paired samples $t$-tests. Statistical significance was assumed at $p \leq 0.05$, and results were based on two-tailed statistical tests. Data were analyzed using SPSS for Windows version 21 (IBM, Armonk, NY, USA).

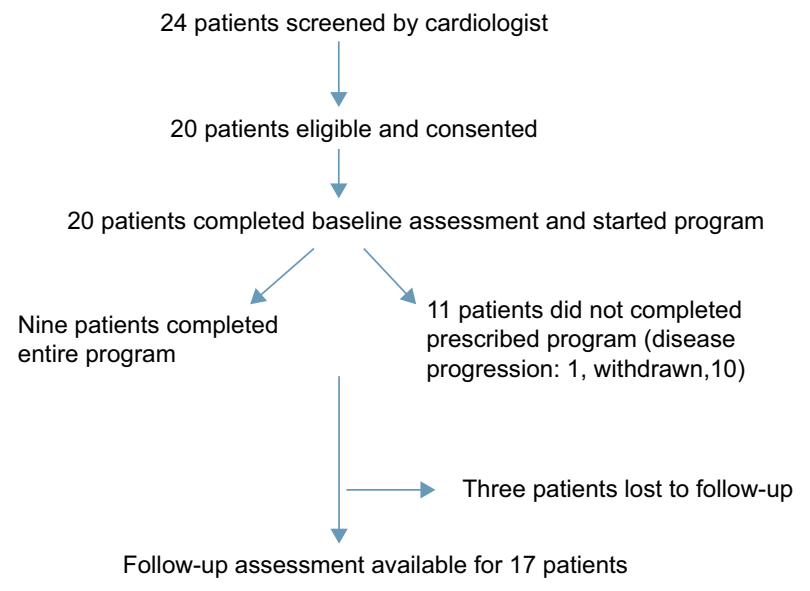

Figure I Flow of patients through the study.
Table I Baseline characteristics $(\mathrm{N}=20)$

\begin{tabular}{ll}
\hline Variable & $\mathbf{n}(\%)$ \\
\hline Age (years) & \\
Mean & 53.1 \\
SD & 8.43 \\
Weight (kg) & \\
Mean & 81.87 \\
SD & 16.19 \\
Marital status & \\
Single & $3(15)$ \\
Married & $13(65)$ \\
Divorced & $3(15)$ \\
Widowed & $1(5)$ \\
Ethnic origin & \\
Caucasian & $17(85)$ \\
Other & $3(15)$ \\
Highest level of education & \\
High school & $2(10)$ \\
College & $10(50)$ \\
University bachelor degree & $5(25)$ \\
University graduate degree & $3(15)$ \\
Systemic chemotherapy & $17(85)$ \\
Adjuvant hormonal therapy & $12(60)$ \\
Trastuzumab & $10(50)$ \\
Radiation & $17(85)$ \\
Neo-adjuvant sequencing of chemotherapy & $4(20)$ \\
Surgery & $19(95)$ \\
Smoking status & \\
Daily & $1(5.0)$ \\
Nonsmoker & $19(95.0)$ \\
\hline Abbrevation: SD, stadard &
\end{tabular}

Abbreviation: SD, standard deviation.

\section{Results}

Twenty women participated in this feasibility study. Descriptive baseline characteristics are presented in Table 1. The mean number of days between surgery and baseline assessment for study enrollment was 301 days (standard deviation [SD] 118) for the 19 patients who had surgery (one patient had a complete clinical response to induction chemoradiation). The median number of days that elapsed between the baseline and follow-up assessment was 241 days (range $=77-490$ days, SD 127). Figure 1 describes the flow of study subjects through the program. The large range is primarily due to differences in times from screening to cardiology consultation, completion of baseline assessment, start of the program at the YMCA, and most significantly, scheduling difficulties regarding availability of the participants to return for their post-study assessment. Several participants did not schedule and attend their follow-up assessment until many months had passed after the end of the program.

\section{Primary outcome measures Accrual}

The collaborating cardiologist approached 24 patients to participate in the trial; however, only 20 provided consent and enrolled. Recruitment efforts were not successful in 
these four individuals for the following reasons: ineligibility $(\mathrm{N}=2$, beyond the age range), did not attend their appointment, and abnormal ECG (patient had ischemic heart disease). The accrual rate was therefore $83 \%$.

\section{Adherence}

Mean adherence to the exercise program was $30.03 \%$ (SD = $38.71 \%$, range $=0.00 \%-95.83 \%$ ). Table 2 indicates adherence for the participants by week.

\section{Accelerometer use}

Patients wore the Actical ${ }^{\circledR}$ device for an average of 68.73 hours over 7 days ( $\mathrm{SD}=35.48$ hours) at baseline and 59.78 hours over 7 days ( $S D=20.14$ hours) at follow-up. Mean number of valid days ( $>10$ hours/day of use) was 3.88 $(\mathrm{SD}=2.33)$ and $3.25(\mathrm{SD}=2.19)$ at baseline and followup, respectively. In other words, the Actical ${ }^{\circledR}$ data capture compliance rate was $78 \%$ at baseline and $65 \%$ at follow-up.

Therefore, the study did not achieve the primary combined outcome (accrual rate over $25 \%$, exercise adherence over $80 \%$, and Actical ${ }^{\circledR}$ data capture compliance over $80 \%$ ) as only the accrual rate was over these predetermined thresholds.

\section{Secondary outcome measures}

Differences in patient-reported outcomes from baseline to follow-up are reported in Table 3.
Table 2 Proportion of participants adhering to their exercise prescription

\begin{tabular}{ll}
\hline Week & $\mathbf{N}(\%)$ \\
\hline 1 & $6(67)$ \\
2 & $5(63)$ \\
3 & $7(88)$ \\
4 & $7(88)$ \\
5 & $6(75)$ \\
6 & $5(71)$ \\
7 & $6(86)$ \\
8 & $3(75)$ \\
9 & $2(50)$ \\
10 & $3(60)$ \\
11 & $3(60)$ \\
12 & $2(40)$ \\
13 & $2(50)$ \\
14 & $3(75)$ \\
15 & $2(50)$ \\
16 & $3(60)$ \\
\hline
\end{tabular}

\section{Fatigue}

A trend towards improvement in the Piper Fatigue Scale was noted. Statistically significant improvement was only found for sensory fatigue.

\section{Quality of life}

Physical, functional, and overall well-being improved on the FACT-B (quality of life).

Table 3 Tests of comparisons between baseline and follow-up for patient-reported parameters

\begin{tabular}{|c|c|c|c|c|c|c|}
\hline & \multirow{2}{*}{$\frac{\text { Baseline }(\mathrm{N}=20)}{\text { Mean (SD) }}$} & \multirow{2}{*}{$\frac{\text { Follow-up }(\mathrm{N}=17)}{\text { Mean }(\mathrm{SD})}$} & \multirow{2}{*}{$\begin{array}{l}\text { Change in score } \\
\text { from baseline }\end{array}$} & \multicolumn{3}{|c|}{ Paired Samples $t$-tests } \\
\hline & & & & $p$ & $t$ & $\eta^{2}$ \\
\hline \multicolumn{7}{|l|}{ Piper Fatigue Scale } \\
\hline Behavioral/severity & $5.3(3.1)$ & $4.3(3.0)$ & -1.00 & 0.11 & 1.69 & 0.15 \\
\hline Affective meaning & $5.6(3.1)$ & $4.8(2.7)$ & -0.77 & 0.17 & 1.45 & 0.11 \\
\hline Sensory & $5.8(2.5)$ & $4.6(2.5)$ & -1.18 & 0.01 & 3.18 & 0.39 \\
\hline Cognitive/mood & $4.8(2.3)$ & $4.8(2.0)$ & -0.05 & 0.92 & 1.00 & 0.00 \\
\hline Total fatigue & $5.4(2.6)$ & $4.6(2.3)$ & -0.73 & 0.09 & 1.80 & 0.17 \\
\hline \multicolumn{7}{|l|}{ FACT-B } \\
\hline Physical well-being & I8.5 (6.I) & $22.3(4.9)$ & +3.82 & 0.00 & -3.73 & 0.46 \\
\hline Social/family well-being & $21.3(6.1)$ & $18.0(6.5)$ & -3.32 & 0.00 & 3.54 & 0.44 \\
\hline Emotional well-being & $17.4(5.2)$ & $18.0(4.3)$ & +0.67 & 0.40 & -0.86 & 0.04 \\
\hline Functional well-being & $15.7(6.3)$ & $19.4(6.1)$ & +3.70 & 0.00 & -4.03 & 0.50 \\
\hline Total well-being & $72.8(18.3)$ & $77.6(17.4)$ & +4.84 & 0.02 & -2.12 & 0.22 \\
\hline \multicolumn{7}{|c|}{ Edmonton Symptom Assessment System } \\
\hline Pain & $2.9(3.3)$ & $2.2(2.3)$ & -0.70 & 0.13 & 1.59 & 0.14 \\
\hline Fatigue & $4.8(3.2)$ & $3.6(2.7)$ & -1.23 & 0.09 & 1.79 & 0.17 \\
\hline Nausea & $0.8(1.8)$ & $0.5(1.7)$ & -0.29 & 0.06 & 2.06 & 0.21 \\
\hline Depression & $2.8(3.4)$ & $1.9(2.7)$ & -0.96 & 0.06 & 1.99 & 0.19 \\
\hline Anxiousness & $3.0(3.5)$ & $2.7(3.1)$ & -0.29 & 0.45 & 0.77 & 0.04 \\
\hline Drowsy & $2.5(2.7)$ & $2.1(2.9)$ & -0.47 & 0.45 & 0.78 & 0.04 \\
\hline Appetite & $1.7(2.1)$ & I.I (2.I) & -0.59 & 0.36 & 0.94 & 0.05 \\
\hline Well-being & $4.4(2.6)$ & $3.5(2.5)$ & -0.88 & 0.07 & 1.95 & 0.19 \\
\hline Shortness of breath & $2.1(3.0)$ & $2.2(2.7)$ & +0.12 & 0.88 & -0.16 & 0.00 \\
\hline
\end{tabular}

Abbreviations: SD, standard deviation; FACT-B, Functional Assessment of Cancer Therapy-Breast. 
Table 4 Tests of comparisons between baseline and follow-up for objective parameters

\begin{tabular}{|c|c|c|c|c|c|c|}
\hline & \multirow{2}{*}{$\begin{array}{l}\text { Baseline }(\mathbf{N}=20) \\
\text { Mean (SD) }\end{array}$} & \multirow{2}{*}{$\begin{array}{l}\text { Follow-up }(\mathrm{N}=\mathrm{I7}) \\
\text { Mean }(\mathrm{SD})\end{array}$} & \multirow{2}{*}{$\begin{array}{l}\text { Change in score } \\
\text { from baseline }\end{array}$} & \multicolumn{3}{|c|}{ Paired Samples t-tests } \\
\hline & & & & $p$ & $t$ & $\eta^{2}$ \\
\hline \multicolumn{7}{|l|}{ Anthropometry } \\
\hline Weight (kg) & $84.3(15.0)$ & $85.9(15.86)$ & +1.6 & 0.31 & -1.06 & 0.07 \\
\hline \multicolumn{7}{|l|}{ Body composition } \\
\hline Fat mass $(\mathrm{kg})$ & $37.6(10.3)$ & $39.2(10.2)$ & +1.56 & 0.23 & -1.26 & 0.09 \\
\hline Lean mass (kg) & $43.5(5.8)$ & $43.8(5.8)$ & +0.37 & 0.35 & -0.97 & 0.06 \\
\hline Percent body fat & $45.8(5.3)$ & $46.8(6.9)$ & +0.95 & 0.45 & -0.76 & 0.04 \\
\hline Percent android fat & $53.5(8.1)$ & $50.6(14.4)$ & +2.92 & 0.39 & 0.88 & 0.05 \\
\hline Visceral adipose tissue $(\mathrm{kg})$ & $\mathrm{I} .5(\mathrm{I} .0)$ & $1.7(1.0)$ & +0.12 & 0.17 & -1.44 & 0.12 \\
\hline Bone mineral content $(\mathrm{kg})$ & $2.4(0.4)$ & $2.4(0.3)$ & -0.08 & 0.02 & 2.71 & 0.31 \\
\hline \multicolumn{7}{|l|}{ Aerobic fitness } \\
\hline Peak $\mathrm{VO}_{2}(\mathrm{~mL} / \mathrm{kg} / \mathrm{min})$ & $20.2(4.7)$ & $20.8(4.6)$ & +0.55 & 0.50 & -0.70 & 0.04 \\
\hline Duration (minutes) & $9.7(2.2)$ & $10.9(1.7)$ & +1.25 & 0.02 & -2.63 & 0.33 \\
\hline Respiratory exchange ratio & I.2(0.I) & I.I (0.I) & -0.02 & 0.46 & 0.77 & 0.05 \\
\hline Pulse at end (beats/min) & I $46.9(24.5)$ & I56.5 (I7.I) & +9.54 & 0.19 & -1.39 & 0.14 \\
\hline Rating of perceived exertion & $15.6(1.4)$ & $16.2(1.9)$ & +0.66 & 0.51 & -0.70 & 0.06 \\
\hline \multicolumn{7}{|l|}{ Physical activity } \\
\hline Sedentary activity (hours) & $5.8(3.7)$ & $14.9(23.3)$ & +9.10 & 0.29 & -1.12 & 0.12 \\
\hline Light-intensity activity (hours) & $1.3(1.0)$ & $3.5(5.3)$ & +2.23 & 0.26 & -1.21 & 0.14 \\
\hline $\begin{array}{l}\text { Moderate- to vigorous-intensity } \\
\text { activity (hours) }\end{array}$ & $2.5(7.4)$ & $0.5(1.0)$ & -2.00 & 0.35 & 0.99 & 0.10 \\
\hline
\end{tabular}

Abbreviations: $\mathrm{SD}$, standard deviation; $\mathrm{VO}_{2}$, oxygen consumption.

Table 5 Frequencies of patient satisfaction responses at follow-up

\begin{tabular}{|c|c|c|c|}
\hline & $\mathbf{n}$ & Mean (SD) & Range \\
\hline Exercise education sessions I attended were helpful & II & $2.18(1.54)$ & $1.00-5.00$ \\
\hline My time as a participant has been enjoyable & 13 & $1.54(0.97)$ & $1.00-4.00$ \\
\hline The program helped me to overcome barriers to being physically active & 13 & $1.77(1.09)$ & $1.00-4.00$ \\
\hline Since becoming a participant, I know more about how to keep physically active & 13 & $2.23(1.17)$ & $1.00-4.00$ \\
\hline The supervised sessions and/or phone calls I received have helped me to become more active & 12 & $2.58(1.38)$ & $1.00-5.00$ \\
\hline The education materials I received were helpful to become more physically active & 11 & $3.00(1.26)$ & $1.00-5.00$ \\
\hline I would tell a friend to become a participant in a program like this & 12 & $1.33(0.89)$ & $1.00-4.00$ \\
\hline I was able to achieve my physical activity goals during the program & 13 & $2.31(1.38)$ & $1.00-5.00$ \\
\hline I am able to do more things I want to do since starting the program & 13 & $2.15(1.21)$ & $1.00-5.00$ \\
\hline I prefer to do physical activity in a group setting & 13 & $2.54(1.13)$ & $1.00-5.00$ \\
\hline I would participate in the program again & 13 & $1.62(1.19)$ & $1.00-5.00$ \\
\hline
\end{tabular}

Abbreviation: SD, standard deviation.

\section{Symptom severity}

There were no significant changes seen in the subscales of the ESAS.

\section{Objective measures}

Secondary objective outcomes can be found in Table 4. Nonsignificant changes were found for weight and objectively measured body composition. BMC significantly decreased from baseline to follow-up. Aerobic fitness variables did not significantly improve over time, except for the duration accomplished for the maximal incremental test. Changes in physical activity levels (hours engaged in sedentary, light, and moderate-to-vigorous activity), as measured by accelerometry, did not significantly change across time.

\section{Patient satisfaction}

Patient satisfaction with the exercise program is indicated in Table 5 (data from 13 respondents who completed this survey). A clear preference for a group program was indicated by 12 respondents. Although comparisons with other populations are difficult, the scores generally are lower than in a previous similar study, for example, an exercise pilot study done with 25 older adults ${ }^{22}$ where item scores were generally between 3.5 and 4.5 out of 5 (Table 5 ).

\section{Discussion}

Given the numerous health benefits afforded by exercise and barriers that breast cancer survivors encounter, the objective of this feasibility study was to comprehensively assess the 
feasibility of a community-based exercise program along with an exploratory examination of the impact of such a program on fatigue levels, quality of life, symptom severity, body composition, aerobic fitness, and physical activity levels. Here, we discuss insights that were gained from our study despite failing to achieve the primary outcome, as well as weaknesses that became apparent retrospectively and may have contributed to this result.

\section{Setting}

Leveraging an existing cardiac rehabilitation program in the community setting, as opposed to a hospital clinic or facility, was a unique feature of this study. As Dittus et al ${ }^{14}$ have reviewed, cardiac rehabilitation is an ideal model because it is widely available and involves risk factor management under the supervision of staff who are trained to identify physical impairments, adapt exercise routines accordingly, and improve fitness. We had hoped that this setting would help foster a return to normalcy for our sample of breast cancer survivors and in turn lead to good adherence rates (a key feature of the composite feasibility end point). However, as noted in the "Results" section, the compound primary end point of feasibility was not met, although accrual initially was adequate. The cardiac rehabilitation community-based exercise program under examination lacked close counseling, motivation sessions, and group cohesion with other cancer survivor participants, which was a weakness whose impact was underestimated during study design. Participants had, by definition, no cardiac conditions and therefore may not have identified as being part of the group of other participants at the YMCA who were cardiac patients and on different exercise prescriptions. In fact, patients agreed in their satisfaction survey that they would have preferred a group-based exercise program with their "peers". Some evidence suggests that supervision is an essential factor for the positive outcomes due to exercise among breast cancer survivors. ${ }^{36}$ In the above-mentioned study by Cheifetz et al, ${ }^{13}$ participants did have formal education sessions during their program specific to cancer survivors which may have contributed to the higher attendance rate in that study. Future investigation should focus not only on accessible exercise facilities but also on building rapport and group cohesion, as well as more actively monitoring and supervising cancer patients to sustain exercise motivation levels.

\section{Timelines and progress through the program}

The high accrual rate $(83 \%)$ indicates that patients were initially highly motivated to explore the use of exercise in combating their fatigue. However, poor adherence rates $(30 \%)$ led to our inability to attain our predefined primary composite end point possibly suggesting motivation to continue waned over time. As outlined in our study, many published exercise programs struggle to attain adherence rates on longer term follow-up $>70 \%{ }^{8-10}$ While breast cancer patients are typically compelled to make lifestyle changes, ${ }^{37,38}$ they are faced with diverse challenges. Obstacles to initiating, adhering, and maintaining an exercise program include the following: employment status; ${ }^{39}$ personal and social resources, ${ }^{40}$ efficacious beliefs, ${ }^{41}$ lack of time, ${ }^{42}$ accessibility to facilities, weakness, and fear of falling, ${ }^{14}$ and lack of priority, ${ }^{43}$ among others.

\section{Adherence and accelerometer use}

In the case of our study, the participants may have been particularly vulnerable to low adherence rates because they were screened for moderate-to-high levels of fatigue (70\% of patients reported high levels of fatigue $[\geq 4 / 10]$ at baseline), which may have affected their energy and motivation levels regarding a physical activity program. In spite of the low adherence rates in the current study, the majority of patients believed that the program enabled them to conquer their exercise-related barriers. It has been suggested that employing generalization of behavior, that is, encouraging patients to exercise beyond the immediate intervention environment, alongside a supervised exercise program, may increase adherence rates. ${ }^{10}$ Furthermore, theory-driven exercise interventions that equip patients with cognitive and behavioral skills, social support, and self-efficacy ${ }^{13,44}$ may also improve adherence rates and overall effectiveness and should be included in future studies of exercise interventions. Accelerometers play an increasingly important role in the objective assessment of physical activity levels, yet it is susceptible to missing data. Although participants in the current study were provided with written and face-to-face verbal instructions, a demonstration on how to wear the Actical ${ }^{\circledR}$ accelerometer, and a monitoring log, compliance at follow-up (post-intervention) proved to be unsatisfactory. Recognizing this methodological challenge, Trost et $\mathrm{al}^{32}$ suggested the following strategies to promote compliance with such devices: reminder calls; tips or lists of frequently asked questions about the accelerometer; devise solutions to overcome barriers associated with accelerometer use (ie., "relapse prevention model"); provide attentiongrabbing materials (eg., posters, magnets, stickers) to serve as reminders; provide participants with an example of the accelerometer data output to encourage accountability; and provide incentives (eg., money, gift certificates, coupons) contingent on compliance. Hence, forthcoming research 
should implement these strategies in an attempt to improve the quality of acquired accelerometry data.

\section{Secondary outcomes}

As this was a feasibility study, the secondary outcomes were examined in an exploratory fashion. No clinically significant changes were evident in most objective outcomes. However, it should be noted that the extreme variability in time between baseline and follow-up assessments possibly obscured intervention effects.

\section{Other studies}

A recent evaluation of the 3-month BEAT Cancer intervention, which involved a supervised moderate-intensity exercise program with counseling, group discussion, and tapering to independent exercise, was conducted. ${ }^{11}$ In contrast to standard care, participants of the BEAT Cancer group significantly improved their aerobic fitness capacity. In accordance with these observations, other community-based exercise programs revealed gains in peak $\mathrm{VO}_{2}$ as well as upper and lower body strength. ${ }^{12-14}$ In the same BEAT Cancer study, Rogers et $\mathrm{al}^{11}$ were the first to exhibit significant improvements in the number of weekly minutes of moderate-intensity physical activity with objective monitoring. In our study, for participants who postponed their follow-up assessment, any gains in aerobic fitness capacity and physical activity patterns due to the intervention may have been diluted as participants may have reverted to their pre-intervention ways.

In the study most similar to ours, Foley and Hasson ${ }^{45}$ examined the effect of an aerobic exercise intervention in 60 breast cancer survivors at a YMCA facility. The adherence was over $85 \%$; however, the participants exercised together in small groups with an instructor, so it was clearly apparent if a participant was not present, a factor the authors consider a key component to the success of their program.

\section{Follow-up and final assessment}

Finally, we should mention the difficulties encountered in regard to timing and follow-up, leading to delays in the completion of the exit assessment which was a vital part of the exploratory (secondary) outcome evaluation. Some patients began their exercise program but requested a temporary break due to personal circumstances that arose (eg., injury, work demands, side effects from comorbidities). Despite returning to the intervention at their readiness, this negatively affected this variable. At times, there was limited communication between the cardiac rehabilitation staff at the YMCA and study personnel regarding the absenteeism from and/or completion of the exercise program, thereby deferring the scheduling of the follow-up assessment. For a handful of patients with poor compliance to the exercise program, repeated attempts were made for them to at least return for their exit appointment, which was therefore delayed. In one case, this took over a year, and although arguably this patient could have been listed as lost to follow-up, we had not set a maximum acceptable delay beforehand and also still wanted to collect satisfaction data. Future studies should focus on incentive and motivational strategies to maintain participants' engagement in the scientific study process, which was perhaps seen as a separate aspect from the exercise instruction at the YMCA (as it was also physically completed at a different location).

\section{Study limitations}

As this was a small feasibility study with mainly Caucasian participants, effect sizes in the secondary outcomes (effects on fatigue, quality of life, and exercise capacity) cannot be generalized. In addition to the small sample size, however, the variability in delays between enrollment, program completion, and follow-up assessment makes it difficult to compare long-term outcomes. It may be that, for example, fatigue improves over time even without intervention. This could only be confirmed with a randomized study with a control arm. In addition, screening of participants for their motivation to adhere to a program may have been useful and should be a part of future studies.

Considering that this was a feasibility study, a control group was not included, which prevents us from inferring causality, and our sample size was relatively small, which affects statistical power and our ability to show significant temporal differences in the secondary end points.

\section{Conclusion}

There is mounting evidence that exercise, in general, is beneficial for individuals along the cancer continuum. However, what is urgently needed are community-based, readily available exercise interventions and recommendations to fit an individual cancer patient's functional ability, disease status, and overall treatment goals. As a feasibility study, this trial demonstrated that a cardiac rehabilitation program can be leveraged for cancer survivors, however that this should likely occur in a cancer survivor group setting rather than on an individual basis within that program. Lack of group cohesiveness and cancer-specific counseling and communication may have lowered participant satisfaction and adversely affected adherence. Successful development and sustaining of an exercise program will be resource-intensive due to the motivational and communication aspects highlighted in 
our study. Although the primary outcome for this study was not achieved, this study contributes valuable insight for the future design and establishment of more generalizable postbreast cancer treatment exercise programs embedded within a cardiac rehabilitation program.

\section{Acknowledgments}

The study was funded by the London Regional Cancer Program and Small Grants Program.

\section{Disclosure}

The authors report no conflicts of interest in this work.

\section{References}

1. International Agency for Research on Cancer, WHO [homepage on the Internet]. GLOBOCAN (2012): estimated cancer incidence, mortality and prevalence worldwide in 2012. Lyon, France: IARC.

2. Torre LA, Bray F, Siegel RL, Ferlay J, Lortet-Tieulent J, Jemal A. Global cancer statistics, 2012. CA Cancer J Clin. 2015;65(2):87-108.

3. Siegel R, Naishadham D, Jemal A. Cancer statistics, 2012. CA Cancer J Clin. 2012;62(1):10-29.

4. Cramp F, Daniel J. Exercise for the management of cancer-related fatigue in adults. Cochrane Database Syst Rev. 2008;(2):CD006145.

5. Barbaric M, Brooks E, Moore L, Cheifetz O. Effects of physical activity on cancer survival: a systematic literature review. Physiother Can. 2010;62(1):25-34.

6. Fong DY, Ho JW, Hui BP, et al. Physical activity for cancer survivors: meta-analysis of randomised controlled trials. BMJ. 2012;344:e70.

7. Meneses-Enchávez JF, González-Jiménez E, Ramírez-Vélez R. Effects of supervised exercise on cancer-related fatigue in breast cancer survivors: a systematic review and meta-analysis. BMC Cancer. 2015;15:77.

8. Courneya KS, Stevinson C, McNeely ML, et al. Predictors of follow-up exercise behavior 6 months after a randomized trial of supervised exercise training in lymphoma patients. Psychooncology. 2012;21(10):1124-1131.

9. Littman AJ, Tang MT, Rossing MA. Longitudinal study of recreational physical activity in breast cancer survivors. J Cancer Surviv. 2010;4(2):119-127.

10. Bourke L, Homer KE, Thaha MA, et al. Interventions to improve exercise behaviour in sedentary people living with and beyond cancer: a systemic review. Br J Cancer. 2014;110(4):831-841.

11. Rogers LQ, Courneya KS, Anton PM, et al. Effects of the BEAT Cancer physical activity behavior change intervention on physical activity, aerobic fitness, and quality of life in breast cancer survivors: a multicenter randomized controlled trial. Breast Cancer Res Treat. 2015;149(1):109-119.

12. Rajotte EJ, Yi JC, Baker KS, Gregersen L, Leiserowitz A, Syrjala KL. Community-based exercise program effectiveness and safety for cancer survivors. J Cancer Surviv. 2012;6(2):219-228.

13. Cheifetz O, Park Dorsay J, Hladysh G, Macdermid J, Serediuk F, Woodhouse LJ. CanWell: meeting the psychosocial and exercise needs of cancer survivors by translating evidence into practice. Psychooncology. 2014;23(2):204-215.

14. Dittus KL, Lakoski SG, Savage PD, et al. Exercise-based oncology rehabilitation: leveraging the cardiac rehabilitation model. J Cardiopulm Rehabil Prev. 2015;35(2):130-139.

15. Mock V. Fatigue management: evidence and guidelines for practice. Cancer. 2001;92(6 Suppl):1699-1707.

16. McGowan E, Prapavessis H, Podolinsky N, Gray C, Elkayam J. The effect of a self-efficacy intervention on objective measures of physical activity in first- and second-degree relatives of colon cancer patients. In: International Psych-Oncology Society 11th World Congress; 21-25 June 2009; Vienna, Austria.
17. Piper BF, Dibble SL, Dodd MJ, Weiss MC, Slaughter RE, Paul SM. The revised Piper Fatigue Scale: psychometric evaluation in women with breast cancer. Oncol Nurs Forum. 1998;25(4):677-684.

18. Cella DF, Tulsky DS, Gray G, et al. The Functional Assessment of Cancer Therapy scale: development and validation of the general measure. J Clin Oncol. 1993;11(3):570-579.

19. Brady MJ, Cella DF, Mo F, et al. Reliability and validity of the Functional Assessment of Cancer Therapy-Breast quality-of-life instrument. J Clin Oncol. 1997;15(3):974-986.

20. Cancer Care Ontario. Edmonton Symptom Assessment System (ESAS). 2005. Available from: https://www.cancercare.on.ca/common/pages/ UserFile.aspx?fileId=13262.

21. Richardson LA, Jones GW. A review of the reliability and validity of the Edmonton Symptom Assessment System. Curr Oncol. 2009;16(1):55.

22. Hooker SP, Seavey W, Weidmer CE, et al. The California active aging community grant program: translating science into practice to promote physical activity in older adults. Ann Behav Med. 2005;29(3):155-165.

23. Madsen OR, Jensen JE, Sørensen OH. Validation of a dual energy X-ray absorptiometer: measurement of bone mass and soft tissue composition. Eur J Appl Physiol Occup Physiol. 1997;75(6):554-558.

24. Wacker WK, Barden HS, Franz JR, Ergun DL. Precision comparison between the Lunar iDXA and Lunar prodigy bone densitometers. J Bone Miner Res. 2006;21(Suppl 1):S355.

25. Balke B, Ware RW. An experimental study of physical fitness in Air Force personnel. U S Armed Forces Med J. 1959;10(6):675-688.

26. Wasserman K, Hansen JE, Sue DY, et al. Measurements during integrative cardiopulmonary exercise testing. In: Wasserman K, Hansen J, Sue D, Stringer W, Whipp B, editors. Principles of Exercise Testing and Interpretation. Baltimore, MD: Lippincott Williams \& Wilkins; 1999:76.

27. Eisenmann JC, Brisko N, Shadrick D, Welsh S. Comparative analysis of the Cosmed Quark b2 and K4b2 gas analysis systems during submaximal exercise. J Sports Med Phys Fitness. 2003;43(2):150-155.

28. Huszczuk A, Whipp BJ, Wasserman K. A respiratory gas exchange simulator for routine calibration in metabolic studies. Eur Respir J. 1990;3(4):465-468.

29. Borg G. Borg's Perceived Exertion and Pain Scales. Stockholm, Sweden: Human Kinetics; 1998.

30. Esliger DW, Copeland JL, Barnes JD, Tremblay MS. Standardizing and optimizing the use of accelerometer data for free-living physical activity monitoring. J Phys Act Health. 2005;3(1):366-383.

31. Esliger DW, Tremblay MS. Physical activity and inactivity profiling: the next generation. Can J Public Health. 2007;98(Suppl 2):S195-S207.

32. Trost SG, McIver KL, Pate RR. Conducting accelerometer-based activity assessments in field-based research. Med Sci Sports Exerc. 2005;37(11 Suppl):S531-S543.

33. Pollock ML, Gaesser GA, Butcher JD, Després JP, Dishman RK, Franklin BA, Garber CE. ACSM position stand: the recommended quantity and quality of exercise for developing and maintaining cardiorespiratory and muscular fitness, and flexibility in healthy adults. Med Sci Sports Exerc. 1998;30(6):975-991.

34. Battaglini C, Bottaro M, Dennehy C, et al. The effects of an individualized exercise intervention on body composition in breast cancer patients undergoing treatment. Sao Paulo Med J. 2007;125(1):22-28.

35. Payne JK, Held J, Thorpe J, Shaw H. Effect of exercise on biomarkers, fatigue, sleep disturbances, and depressive symptoms in older women with breast cancer receiving hormonal therapy. Oncol Nurs Forum. 2008;35(4):635-642.

36. Velthuis MJ, Agasi-Idenburg SC, Aufdemkampe G, Wittink HM. The effect of physical exercise on cancer-related fatigue during cancer treatment: a meta-analysis of randomised controlled trials. Clin Oncol (R Coll Radiol). 2010;22(3):208-221.

37. Demark-Wahnefried W, Peterson B, McBride C, Lipkus I, Clipp E. Current health behaviors and readiness to pursue life-style changes among men and women diagnosed with early stage prostate and breast carcinomas. Cancer. 2000;88(3):674-684.

38. Wayne SJ, Lopez ST, Butler LM, Baumgartner KB, Baumgartner RN, Ballard-Barbash R. Changes in dietary intake after diagnosis of breast cancer. J Am Diet Assoc. 2004;104(10):1561-1568. 
39. Steinhilper L, Geyer S, Sperlich S. Health behavior change among breast cancer patients. Int J Public Health. 2013;58(4):603-613.

40. Aspinwall, LG. Dealing with adversity: self-regulation, coping, adaptation, and health. In: Brewer MB, Hewstone M, editors. Applied Social Psychology. Malden, MA: Blackwell; 2004:3-27.

41. Sherman KA, Heard G, Cavanagh KL. Psychological effects and mediators of a group multi-component program for breast cancer survivors. J Behav Med. 2010;33(5):378-391.

42. Haas BK, Kimmel G, Hermanns M, Deal B. Community-based FitSTEPS for life exercise program for persons with cancer: 5-year evaluation. J Oncol Pract. 2012;8(6):320-324.
43. Basen-Engquist K, Taylor CL, Rosenblum C, et al. Randomized pilot test of a lifestyle physical activity intervention for breast cancer survivors. Patient Educ Couns. 2006;64(1-3):225-234.

44. Rogers LQ, Courneya KS, Shah P, Dunnington G, Hopkins-Price P. Exercise stage of change, barriers, expectations, values and preferences among breast cancer patients during treatment: a pilot study. Eur $J$ Cancer Care (Engl). 2007;16(1):55-66.

45. Foley MP, Hasson SM. Effects of a community-based multimodal exercise program on health-related physical fitness and physical function in breast cancer survivors: a pilot study. Integr Cancer Ther. 2016;15(4):446-454.
Cancer Management and Research

\section{Publish your work in this journal}

Cancer Management and Research is an international, peer-reviewed open access journal focusing on cancer research and the optimal use of preventative and integrated treatment interventions to achieve improved outcomes, enhanced survival and quality of life for the cancer patient The manuscript management system is completely online and includes

\section{Dovepress}

a very quick and fair peer-review system, which is all easy to use. Visit $\mathrm{http}: / /$ www.dovepress.com/testimonials.php to read real quotes from published authors. 